\title{
First passages in bounded domains: When is the mean first passage time meaningful?
}

\author{
Thiago G. Mattos* \\ Max Planck Institute for Intelligent Systems, Heisenbergstraße 3, 70569 Stuttgart, Germany \\ Carlos Mejía-Monasterio \\ Laboratory of Physical Properties, Technical University of Madrid, Avenida Complutense s/n, 28040 Madrid, Spain and \\ Department of Mathematics and Statistics, University of Helsinki, P.O. Box 68, FIN-00014 Helsinki, Finland \\ Ralf Metzler \\ Institute for Physics \& Astronomy, University of Potsdam, 14476 Potsdam-Golm, Germany and \\ Physics Department, Tampere University of Technology, Korkeakoulunkatu 3, FIN-33101 Tampere, Finland \\ Gleb Oshanin \\ Laboratoire de Physique Théorique de la Matière Condensée (UMR CNRS 7600), Université Pierre et Marie Curie (Paris 6), \\ 4 Place Jussieu, 75252 Paris, France
}

(Received 7 August 2012; published 27 September 2012)

\begin{abstract}
We study the first passage statistics to adsorbing boundaries of a Brownian motion in bounded two-dimensional domains of different shapes and configurations of the adsorbing and reflecting boundaries. From extensive numerical analysis we obtain the probability $P(\omega)$ distribution of the random variable $\omega=\tau_{1} /\left(\tau_{1}+\tau_{2}\right)$, which is a measure for how similar the first passage times $\tau_{1}$ and $\tau_{2}$ are of two independent realizations of a Brownian walk starting at the same location. We construct a chart for each domain, determining whether $P(\omega)$ represents a unimodal, bell-shaped form, or a bimodal, M-shaped behavior. While in the former case the mean first passage time (MFPT) is a valid characteristic of the first passage behavior, in the latter case it is an insufficient measure for the process. Strikingly we find a distinct turnover between the two modes of $P(\omega)$, characteristic for the domain shape and the respective location of absorbing and reflective boundaries. Our results demonstrate that large fluctuations of the first passage times may occur frequently in two-dimensional domains, rendering quite vague the general use of the MFPT as a robust measure of the actual behavior even in bounded domains, in which all moments of the first passage distribution exist.
\end{abstract}

DOI: 10.1103/PhysRevE.86.031143

PACS number(s): 02.50.-r, 03.65.Nk, 42.25.Dd, 73.23.-b

\section{INTRODUCTION}

The concept of first passage underlies diverse stochastic processes in which it is relevant when the value of the random variable reaches a preset value for the first time. A few stray examples across disciplines include chemical reactions [1-6], the firing of a neuron [7,8], random search of a mobile or immobile target [9-19], diffusional disease spreading [20], DNA bubble breathing [21], dynamics of molecular motors $[22,23]$, and the triggering of a stock option [24]. A variety of first passage time phenomena and different related results have been investigated in Refs. [25,26]. While for continuous processes the first passage across a given preset value coincides with the first arrival to exactly this value, for Lévy flights characterized by long-tailed jump length distributions with diverging variance both quantities become different, and large overshoots across a preset value occur [27].

The distribution of first passage times in unbounded domains is typically broad, such that not even the mean first passage time exists [26]. In particular, in one-dimensional, semi-infinite domains the first passage time distribution of a Markovian process is universally dominated by the $t^{-3 / 2}$ scaling nailed down by the Sparre Andersen theorem [26]. A similar divergence of the mean first passage time occurs in

*Corresponding author; tgmattos@is.mpg.de stochastic processes characterized by scale-free distributions of waiting times [28]. In contrast, in many practically important situations first passage processes involve particles which move randomly in bounded domains (see, e.g., Refs. [29-32]). In this case the random variable of interest, the first passage time $\tau$ to, e.g., a boundary, a target chemical group, or a binding site on the surface of the domain or elsewhere within the domain has a distribution $\Psi(\tau)$ of the generic, generalized inverse Gaussian form (see, e.g., the discussion in Ref. [15] and references therein)

$$
\Psi(\tau) \sim \exp \left(-\frac{a}{\tau}\right) \frac{1}{\tau^{1+\mu}} \exp \left(-\frac{\tau}{b}\right)
$$

where $a$ and $b$ are some constants dependent on the shape of the domain, the exact starting point within the domain, etc., and $\mu$ is the so-called persistence exponent [33]. When the linear size of the domain (say, the radius $R$ of a circular or a spherical domain) diverges (i.e., $R \rightarrow \infty$ ), the parameter $b$ also diverges such that the long-time asymptotic behavior of the first passage time distribution is of power-law form without a cutoff. In this case, at least some, if not all, of the moments of $\Psi(\tau)$ diverge.

The first passage time distribution in Eq. (1) is exact only in the particular case of Brownian motion on a semi-infinite line in the presence of a bias pointing towards the target site, or, equivalently, for the celebrated integrate-and-fire model 
of neuron firing by Gerstein and Mandelbrot [7]. In general, the detailed form of $\Psi(\tau)$ is obviously much more complex than given by Eq. (1), depending on the very shape of the domain under consideration and the exact boundary value problem. Typically $\Psi(\tau)$ is given in terms of an infinite series. Nonetheless, on a qualitative level, the approximation (1) provides a clear picture of the actual behavior of the first passage time distribution in bounded domains. Namely, $\Psi(\tau)$ consists of three different parts: a singular decay for small values of $\tau$, which mirrors the fact that the first passage to some point starting from a distant position cannot occur instantaneously. This is followed at intermediate times by a generic power-law decay with exponent $\mu$, depending on the exact type of random motion. Finally, an exponential decay at long $\tau$ cuts off the power law. A crucial aspect is that the exponential cutoffs at both short and long $\tau$ ensure that in bounded domains $\Psi(\tau)$ possesses moments of arbitrary positive or negative order.

Distributions of the form (1) are usually considered narrow, as opposed to broad distributions, which do not possess all moments [25-28], e.g., $\Psi(\tau)$ in Eq. (1) with $b=\infty$. Once all moments exist, it is often tacitly assumed that the first moment of this distribution, the mean first passage time (MFPT)

$$
\langle\tau\rangle=\int_{0}^{\infty} \tau \Psi(\tau) d \tau,
$$

is an adequate measure of the first passage behavior. The actual analytical calculation of the MFPT may require a considerable computational effort, and the calculation of higher moments is quite formidable and is not always possible (see, e.g., Refs. [29-31]). Conversely, it has been demonstrated in, e.g., recent Refs. [34-39] that random variables with truncated power-law distributions behave in several important aspects as those characterized by nontruncated, broad distributions, revealing substantial fluctuations between individual realizations and thus rendering the concept of a mean first passage time a bit unsubstantiated. To be more precise, this concerns not the functional form of the MFPT for a given process, but rather its use as a characteristic quantity for the process. The functional form of the MFPT is certainly an important property, providing valuable insights to the scaling behavior, for instance with the system size or the initial distance of starting point and target. In contrast, the very numerical value of the MFPT can significantly differ from the values drawn from individual trajectories. Therefore, the MFPT can be substantially larger than the most probable value for the first passage time. Clearly, an understanding of how representative the MFPT is of the actual behavior and, concurrently, how important fluctuations of $\tau$ between individual realizations indeed are is of the utmost conceptual importance in many areas, such as an interpretation of the first passage data obtained from single-particle tracking.

In this paper we analyze, via extensive Monte Carlo simulations, the role of fluctuations between individual realizations of first passage times for Brownian motion (BM) in two-dimensional bounded domains of different shapes, and with different configurations of the reflective and adsorbing boundaries. Analogous results for three-dimensional systems and for systems with quenched disorder will be presented elsewhere [40].

\section{SIMULTANEITY CONCEPT OF FIRST PASSAGE}

To quantify the relevance of such fluctuations and the effective broadness of the corresponding first passage time distribution $\Psi(\tau)$ we employ a diagnostics method based on the concept of simultaneity of first passage events; see Fig. 1. Instead of the original first passage problem with quantifying the statistical outcome for a single Brownian walker, we simultaneously launch two identical, noninteracting Brownian particles at the same position $\mathbf{r}_{\mathbf{0}}$ (which is identical to two different realizations of the trajectories of a single BM starting at $\mathbf{r}_{\mathbf{0}}$ ). The corresponding outcomes are the first passage times $\tau_{1}$ and $\tau_{2}$. We now define the random variable

$$
\omega \equiv \frac{\tau_{1}}{\tau_{1}+\tau_{2}}
$$

such that $\omega$ ranges in the interval $[0,1]$. The uniformity index $\omega$ measures the likelihood that both walkers arrive at the adsorbing boundary simultaneously: When $\omega$ is close to $1 / 2$, the process is uniform. In contrast, values of $\omega$ close to 0 or 1 mean highly nonuniform behavior, implying that the MFPT is not representative of the actual behavior, but is merely the first moment of an effectively broad distribution. We note parenthetically that similar random variables have been used in the analysis of random probabilities induced by normalization of self-similar Lévy processes [41], of the fractal characterization of Paretian Poisson processes [42], and of the so-called matchmaking paradox $[43,44]$.

Within a given bounded domain we evaluate the distribution $P(\omega)$ measuring the uniformity of the first passage dynamics with respect to some fixed starting point $\mathbf{r}_{\mathbf{0}}$. This is repeated for a large number of nodes $\mathbf{r}_{\mathbf{0}}$ within the domain, thus producing a uniformity chart of first passage. Remarkably, we find that the very shape of this distribution depends delicately on the domain shape, the actual settings of adsorbing and reflecting boundaries, and on the starting location $\mathbf{r}_{\mathbf{0}}$. In some starting areas $P(\omega)$ has a characteristic unimodal, bell-shaped form with a maximum at $\omega=1 / 2$, signaling that most pairs of BMs will arrive at the adsorbing boundary simultaneously. This means, in turn, that in this case the parental first passage time distribution $\Psi(\tau)$ can be considered as sufficiently narrow such that the MFPT can be considered as a plausible measure

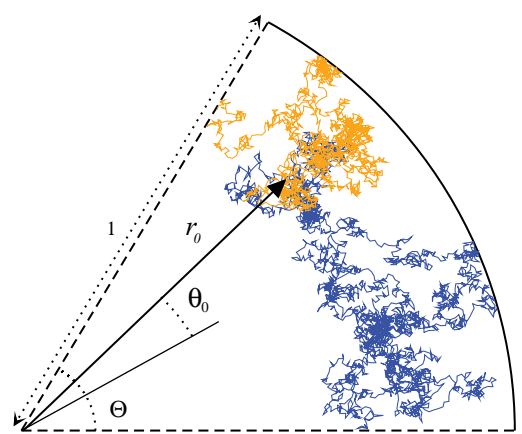

FIG. 1. (Color online) Trajectories of two Brownian walkers starting at the same initial position $\left(r_{0}, \theta_{0}\right)$ inside a bounded pie-wedge domain with opening angle $\Theta$ as well as absorbing radial boundaries (dashed lines) and reflecting boundary (solid line) at $r=1$. The values of the first passage times to the adsorbing boundaries are used to construct the random variable $\omega$. 
of individual first passage events, providing a rather accurate estimate for the typical value of the first passage time. Conversely, we find that for other starting areas $P(\omega)$ exhibits a completely different behavior and has a characteristic bimodal, M-shaped form with a local minimum at $\omega=1 / 2$ and two maxima close to $\omega=0$ and $\omega=1$. In that case simultaneous arrival of two initially synchronized walkers is unlikely; i.e., any two trajectories will most likely possess distinctly different first passage times. The parental first passage time distribution $\Psi(\tau)$ is consequently broad and sample-to-sample fluctuations matter: The MFPT cannot be considered as an adequate measure of the actual behavior. Given that, by definition, the averages $\left\langle\tau_{1}\right\rangle$ and $\left\langle\tau_{2}\right\rangle$ are identical and, moreover, the moments of $\tau_{1}$ and $\tau_{2}$ of arbitrary order coincide, one can think of $\omega$ [and, hence, of the distribution $P(\omega)$ ] as a measure of the symmetry breaking between different realizations of the process. Note also that situations in which the mean value of some pertinent parameter is dominated by the tails of the distribution, and this mean thus has a very different value compared to the most probable value (and may even show a completely different dependence on the system parameters), is most often encountered in disordered systems [18,39]. Here we observe such a behavior in the absence of any disorder.

Scanning then over the possible starting points within each bounded domain, we obtain a corresponding phase chart for $P(\omega)$, distinguishing regions in which $P(\omega)$ has M-shaped or bell-shaped behavior. The demarcation zone between these two phases, depicted by beige color in the following, represents a plateau-like, almost uniform behavior of $P(\omega)$ with zero second derivative at $\omega=1 / 2$.

We proceed by giving a general definition of the first passage time distribution $\Psi(\tau)$ and its corresponding MFPT in Sec. III, and also establish a relation between $\Psi(\tau)$ and the uniformity distribution $P(\omega)$. In Sec. IV we study in detail the problem of Brownian motion in a pie-wedge-shaped domain with absorbing and reflecting boundaries. In Secs. V and VI we discuss the forms of $P(\omega)$, as a function of the location of the starting point, for circular domains with small aperture on the boundary, a two-dimensional version of the so-called narrow escape time problem [48], and for triangular domains with adsorbing boundaries, respectively. Our results are summarized in Sec. VII.

\section{FIRST PASSAGE DISTRIBUTION, MEAN FIRST PASSAGE TIME, AND THE UNIFORMITY DISTRIBUTION $P(\omega)$}

Consider a BM inside a general two-dimensional domain $\mathcal{S}$, whose boundary $\partial \mathcal{S} \equiv \partial \mathcal{S}_{a} \cup \partial \mathcal{S}_{r}$ comprises reflecting, $\partial \mathcal{S}_{r}$, and absorbing, $\partial \mathcal{S}_{a}$, parts. At time $t=0$, the BM initiates at $\mathbf{r}_{0} \in \mathcal{S}$ and evolves within the domain until the trajectory hits $\partial \mathcal{S}_{a}$ for the first time at some random instant $\tau$. Furthermore let $P\left(\mathbf{r}, t \mid \mathbf{r}_{0}\right)$ denote the conditional probability distribution for finding the Brownian walker at position $\mathbf{r}$ at time $t$, provided the initial condition was at $\mathbf{r}_{0}$ at $t=0$. The distribution $P\left(\mathbf{r}, t \mid \mathbf{r}_{0}\right)$ is the solution of the diffusion equation

$$
\frac{\partial}{\partial t} P\left(\mathbf{r}, t \mid \mathbf{r}_{0}\right)=D \nabla_{\mathbf{r}}^{2} P\left(\mathbf{r}, t \mid \mathbf{r}_{0}\right)
$$

on $\mathcal{S}$, where $\nabla_{\mathrm{r}}^{2}$ is the two-dimensional Laplacian equivalent to $\partial^{2} / \partial x^{2}+\partial^{2} / \partial y^{2}$ in Cartesian coordinates. Equation (4) is subject to the initial condition as well as the boundary conditions at $\partial \mathcal{S}$. Here $D$ is the diffusion coefficient. The solution of this boundary value problem is, in the best case, cumbersome, and explicit solutions may be obtained for only a few simple geometries; see Ref. [45].

If a finite part of the boundary is absorbing, i.e., $\partial \mathcal{S}_{a}$ is not empty, then the distribution $P\left(\mathbf{r}, t \mid \mathbf{r}_{0}\right)$ is no longer normalized. The survival probability $\mathscr{S}_{\mathbf{r}_{0}}(t)$ that the walker has not reached $\partial \mathcal{S}_{a}$ up to time $t$ is defined by

$$
\mathscr{S}_{\mathbf{r}_{0}}(t)=\int_{\mathcal{S}} P\left(\mathbf{r}, t \mid \mathbf{r}_{0}\right) d \mathbf{r}
$$

$\mathscr{S}_{\mathbf{r}_{0}}(t)$ is a monotonically decreasing function of time, eventually reaching zero value, $\lim _{t \rightarrow \infty} \mathscr{S}_{\mathbf{r}_{0}}(t)=0$. The desired distribution of first passage times to the adsorbing boundary becomes

$$
\Psi_{\mathbf{r}_{0}}(\tau)=-\frac{d \mathscr{S}_{\mathbf{r}_{0}}(\tau)}{d \tau} .
$$

The MFPT associated with the distribution $\Psi(\tau)$ is defined as the first moment

$$
\langle\tau\rangle\left(\mathbf{r}_{0}\right)=\int_{0}^{\infty} \tau \Psi_{\mathbf{r}_{0}}(\tau) d \tau=\int_{0}^{\infty} \mathscr{S}_{\mathbf{r}_{0}}(\tau) d \tau .
$$

We note parenthetically that in most of the existing literature, apart from recent Refs. [3,29,30], the dependence of the MFPT on the starting position of the walker is either simply neglected, or it is assumed that the starting point is randomly distributed within the domain $\mathcal{S}$. As we proceed to show, the $\mathbf{r}_{0}$ dependence of the first passage time distribution is a crucial aspect which cannot be neglected.

We now turn to the uniformity distribution $P(\omega)$ of the random variable $\omega$, Eq. (3). Let

$$
\Phi(\lambda)=\int_{0}^{1} P(\omega) \exp (-\lambda \omega) d \omega,
$$

with $\lambda \geqslant 0$, denote the moment generating function of $\omega$. Since $\tau_{1}$ and $\tau_{2}$ are independent, identically distributed random variables, expression (8) can formally be represented as

$$
\Phi(\lambda)=\int_{0}^{\infty} \int_{0}^{\infty} \Psi\left(\tau_{1}\right) \Psi\left(\tau_{2}\right) \exp \left(-\lambda \frac{\tau_{1}}{\tau_{1}+\tau_{2}}\right) d \tau_{1} d \tau_{2} .
$$

Integrating over $d \tau_{1}$ we change the integration variable, $\tau_{1} \rightarrow$ $\omega$, so that Eq. (9) is rewritten in the form

$$
\begin{aligned}
\Phi(\lambda)= & \int_{0}^{1} \exp (-\lambda \omega) \frac{d \omega}{(1-\omega)^{2}} \\
& \times \int_{0}^{\infty} \tau_{2} \Psi\left(\tau_{2}\right) \Psi\left(\frac{\omega}{1-\omega} \tau_{2}\right) d \tau_{2} .
\end{aligned}
$$

From comparison with Eq. (8), we readily read off the desired distribution function

$$
P(\omega)=\frac{1}{(1-\omega)^{2}} \int_{0}^{\infty} \tau \Psi(\tau) \Psi\left(\frac{\omega}{1-\omega} \tau\right) d \tau .
$$

Therefore, $P(\omega)$ is known for given $\Psi(t)$.

To get an idea of the typical behavior of the uniformity distribution $P(\omega)$, we use the generic form (1) for the 
first passage time distribution. From Eq. (11) we find from integration that

$$
P(\omega)=\frac{1}{2 K_{\mu}^{2}\left(2 \sqrt{\frac{a}{b}}\right)} \frac{1}{\omega(1-\omega)} K_{2 \mu}\left(2 \sqrt{\frac{a}{b \omega(1-\omega)}}\right),
$$

where $K_{2 \mu}(\cdot)$ is the modified Bessel function of the second type. It was realized $[15,38]$ that the form of the distribution $P(\omega)$ in Eq. (12) is distinctly sensitive to the value of the persistence exponent $\mu$, which characterizes the scaling behavior of the first passage time distribution $\Psi(\tau)$ at intermediate times. Thus, for $\mu>1, P(\omega)$ is always a unimodal, bell-shaped function with a maximum at $\omega=1 / 2$. For $\mu=1, P(\omega)$ is almost uniform, $P(\omega) \approx 1$, apart from narrow regions at the corners $\omega=0$ and $\omega=1$, for $b / a \gg 1$. Curiously, for $\mu<1$, which corresponds to the most common case, there exists a critical value $p_{c}$ of the ratio $p=b / a$ such that for $p>p_{c}$ the distribution $P(\omega)$ has a characteristic M-shaped form with two maxima close to 0 and 1 , while at $\omega=1 / 2$ we find a local minimum. Such a transition from a unimodal, bell-shaped to bimodal, M-shaped form mirrors a significant manifestation of sample-to-sample fluctuations that has been indeed observed in exact calculations of $P(\omega)$ for Brownian search processes for an immobile target in $d$-dimensional spherical geometries [15].

In what follows we further explore this intriguing behavior of the first passage time distribution via extensive Monte Carlo simulations focusing on the effects of the domain shape, the type of the boundary conditions, and the initial position of the walker.

\section{UNIFORMITY DISTRIBUTION $P(\omega)$ IN A PIE-WEDGE DOMAIN}

Consider now the case of a bounded domain of pie-wedge shape with unit radius $R=1$ and opening angle $\Theta$. The absorbing boundaries correspond to the radial edges, while the outer circular edge is reflective; see Fig. 1. Clearly, for a $\mathrm{BM}$ inside such a pie-wedge domain, all moments of the first passage time distribution exist.

Before we proceed to investigate this case we first turn to the case when the wedge radius is infinite, $R \rightarrow \infty$. Then the distribution function $P\left(\mathbf{r}, t \mid \mathbf{r}_{0}\right)$ is known exactly (see, e.g., Refs. [26,46]) and is represented by an infinite series whose leading term for $t \rightarrow \infty$ is given, up to a normalization constant, by

$$
P\left(\mathbf{r}, t \mid \mathbf{r}_{0}\right) \simeq \frac{\pi \sin \left(\pi \theta_{0} / \Theta\right)}{4 D \Theta t} e^{-\left(\rho^{2}+\rho_{0}^{2}\right) / 4 D t} I_{\pi / \Theta}\left(\frac{\rho_{0} \rho}{2 D t}\right),
$$

where $I_{v}(z)$ is the modified Bessel function of the first kind and $\mathbf{r}=(\rho, \theta)$ is conveniently represented in polar coordinates. This solution is obtained for the sharp initial condition $P\left(\mathbf{r}, 0 \mid \mathbf{r}_{0}\right)=\pi \sin \left(\pi \theta_{0} / \Theta\right) \delta\left(\mathbf{r}-\mathbf{r}_{\mathbf{0}}\right) / 2 \Theta \rho_{0}$. From Eq. (13) one finds the asymptotic behavior of the survival probability,

$$
\mathscr{S}_{\mathbf{r}_{0}}(t) \simeq\left(\frac{\rho_{0}^{2}}{D}\right)^{\pi / 2 \Theta} t^{-\pi / 2 \Theta},
$$

such that the first passage time distribution becomes

$$
\Psi_{\mathbf{r}_{0}}(t) \simeq \frac{\pi}{2 \Theta}\left(\frac{\rho_{0}^{2}}{D}\right)^{\pi / 2 \Theta} \frac{1}{t^{1+\pi / 2 \Theta}}
$$

Note that this distribution is of the generic form (1), where $b=$ $\infty$ due to the infinite domain size. The nonuniversal persistence exponent is given by

$$
\mu=\frac{\pi}{2 \Theta}
$$

Therefore, the MFPT diverges when $\Theta \geqslant \pi / 2$ and is finite for $\Theta<\pi / 2$. According to the qualitative analysis from Sec. III, $P(\omega)$ will have a bimodal form in the former case and a unimodal one in the latter.

We now turn our attention to finite-sized pie wedges, for which the MFPT and all higher moments of Eq. (6) are finite. In principle, an exact solution for the first passage time distribution in this case can be obtained by solution of the corresponding mixed boundary value problem, but the result will be too cumbersome for our purposes. Instead, we resort to numerical simulations. We now show that for finite pie wedges the actual behavior is in fact richer than in the case of an infinite wedge.

We performed Monte Carlo simulations of a random walk inside a pie wedge of unit radius and opening angle $\Theta$. The boundary conditions along the radii are absorbing and reflecting along the circular edge; see Fig. 1. The random walk is simulated in terms of a standard Pearson walk on a plane (compare Ref. [47]), which consists of a sequence of steps of fixed length $\lambda=0.001$ and uniform waiting time $v=1 / \lambda$. After each step the walker turns by a random angle with uniform distribution. At time $t=0$ the walker is released at $\left(\rho_{0}, \theta_{0}\right)$, and its trajectory is recorded until it hits a point on the absorbing boundary for the first time. Generating $N$ (we used $N=10^{5}$ ) such trajectories, we obtain a set of first passage times $\left\{\tau_{i}\right\}$, from which we construct the first passage time distribution. Since all $\tau_{i}$ are independent, identically distributed random variables, the uniformity distribution $P(\omega)$ is then readily obtained via Eq. (3) from distinct pairs $\tau_{1}$ and $\tau_{2}$ chosen at random from the set $\left\{\tau_{i}\right\}$.

Figure 2(a) shows the first passage time distributions corresponding to a fixed $\rho_{0}=0.76$ and three different starting angles $\theta_{0}$ for a pie wedge with opening angle $\Theta=\pi / 2$. One notices that, for small and large values of $\tau$, all three distributions $\Psi(\tau)$ significantly deviate from the intermediate power-law behavior, which is due to exponential tempering. On the other hand, at intermediate times the distributions exhibit a slower, power-law like decay within a range that depends significantly on $\theta_{0}$. The narrowest distribution (light blue) is obtained for a starting position $(0.76,0)$, which is exactly on the symmetry axis of the wedge. Increasing the angle $\theta_{0}$ away from the symmetry axis results in a broadening of $\Psi(\tau)$, and the intermediate algebraic decay is more pronounced.

In panel (b) of Fig. 2 we plot the corresponding uniformity distributions $P(\omega)$. For the narrowest first passage time distribution $\Psi(\tau)$ (light blue symbols), $P(\omega)$ is bell shaped with $1 / 2$ representing the most probable value, such that sample-to-sample fluctuations of $\tau$ are less significant. In this case, apparently, the MFPT is a meaningful, reliable measure of the first passage behavior, and any two walkers starting from the same position on the symmetry axis of the wedge will most likely be absorbed at the same instant of time. Strikingly, we find that this is no longer valid for the two other starting 

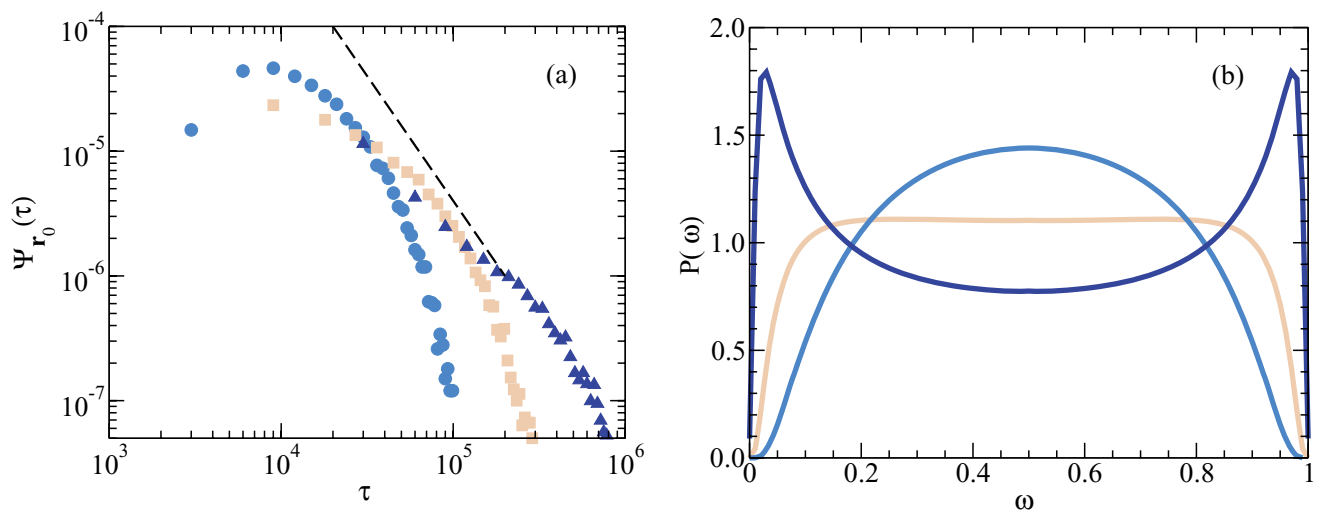

FIG. 2. (Color online) (a) First passage time distribution $\Psi_{\left(\rho_{0}, \theta_{0}\right)}(\tau)$ for a Brownian walk in a pie-wedge domain with opening angle $\Theta=\pi / 2$. Different colors and symbols correspond to different starting points: $\left(\rho_{0}=0.76, \theta_{0}=-0.38 \Theta\right)($ dark blue/triangles $),(0.76,-0.23 \Theta)$ (beige/squares), and $(0.76,0)$ (light blue/circles). The dashed straight line indicates the intermediate power-law decay $\Psi(\tau) \sim 1 / \tau^{2}$, Eq. $(15)$. (b) The corresponding distribution $P(\omega)$ with the same color and symbol coding. In grey scale, dark blue corresponds to the darkest shade of grey, light blue to dark grey, and beige to light grey.

positions off the symmetry axis: For $\mathbf{r}_{\mathbf{0}}=(0.76,-\pi / 3)$ the uniformity distribution $P(\omega)$ is almost uniform, except for narrow regions in the vicinity of the edges, meaning that any relation between the first passage times of two walkers is equally probable. Finally, for starting position $(0.76,-\pi / 7)$, which is the one closest to the absorbing boundary, $P(\omega)$ has a characteristic M-shaped form with maxima close to 0 and 1 , and a local minimum at $\omega=1 / 2$. This signifies that in such a case the symmetry between any two walkers is most distinctly broken, and they will be absorbed at very different times. Clearly, in this case the MFPT is not representative of the actual behavior.

To quantify the shape of the distribution of $\omega$ we perform a fit of the numerically obtained $P(\omega)$ to a quadratic polynomial of $\omega$ in the domain $0.05<\omega<0.95$. From this fit we obtain the coefficient $\chi$ of the quadratic term. The sign of $\chi$ thus determines the shape of $P(\omega): \chi<0$ corresponds to the unimodal, bell-shaped distribution; $\chi>0$ signifies that the distribution is bimodal, M-shaped; and a zero value of $\chi=0$ means that $P(\omega)$ is uniform. Such a procedure, of course, has some ambiguities, especially when we deal with the demarcation line $\chi=0$ between regions in which $P(\omega)$ has unimodal and bimodal forms, as it is not always clear how many digits are to be taken into account. This results in a certain broadening of the demarcation line. However, we have checked in several cases that this procedure produces reliable results. In Fig. 3 we show the evolution of $\chi$ versus the starting angle $\theta_{0}$ for fixed $\rho_{0}=0.76$ in a pie-wedge domain with the opening angle $\Theta=\pi / 2$. We observe a continuous, periodic variation of $P(\omega)$ with the starting position, changing from an M-shaped to a bell-shaped form. The insets of Fig. 3 show the schematic distribution $P(\omega)$ for some specific values of $\theta_{0}$. The absolute value of $\chi$ indicates how far the distribution $P(\omega)$ deviates from a locally uniform distribution.

Finally, we used this approach to create the phase chart for the shape of the uniformity distribution $P(\omega)$ with respect to the starting position of the walker within the pie-wedge domain. In Fig. 4 we present a systematic scan of the domain for three pie-wedges with different opening angles.
One observes that in all three cases, there exists a region in which $P(\omega)$ is bell shaped (light blue symbols) and a region with $\mathrm{M}$-shaped $P(\omega)$ (dark blue symbols), separated by a small region with nearly uniform distribution (beige symbols).

Therefore, as we have already remarked, the actual behavior in a finite pie wedge appears to be much richer than in an infinite wedge. Consider an experiment in which one aims to find an estimate of the MFPT by tracking the evolution of a few single-particle trajectories starting at the same position inside the light blue region. The outcome of such an experiment will be a good estimate of the MFPT, with reliably small error. This will be the case since in the light blue region $P(\omega)$ is bell shaped, which means that the probability that the two trajectories arrive at the same time is maximal. In contrast, if two single-particle trajectories start anywhere inside the dark blue region, then it is most likely that these trajectories will arrive at the adsorbing boundary at very different times, yielding a poor and unreliable estimate for the MFPT. The sample-to-sample fluctuations in this case are very important

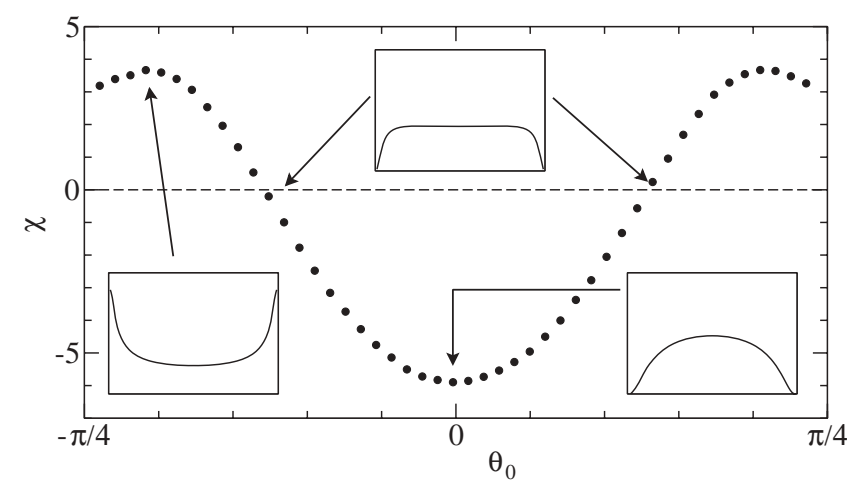

FIG. 3. Dependence of the parameter $\chi$ on the starting angle $\theta_{0}$ for fixed $\rho_{0}=0.76$ in a pie-wedge domain with opening angle $\Theta=\pi / 2$. The three insets show the shape of the uniformity distribution $P(\omega)$ for the $\theta_{0}$ values indicated by the arrows. 


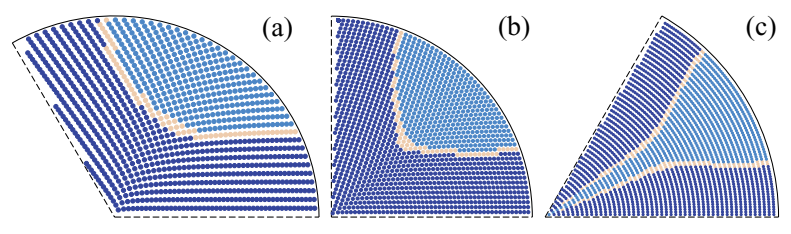

FIG. 4. (Color online) Phase chart for the shape of the uniformity distribution $P(\omega)$ in three different pie-wedge domains: (a) $\Theta=$ $3 \pi / 4$, (b) $\Theta=\pi / 2$, (c) $\Theta=\pi / 3$. The color of the symbols is light blue if $\chi<-\chi_{\star}$, dark blue if $\chi>\chi_{\star}$, and beige if $|\chi|<\chi_{\star}$, where we chose $\chi_{\star}=0.25$. For each initial location, $P(\omega)$ was computed from a sample of $N=10^{5}$ random trajectories.

and, as a consequence, the MFPT is not an adequate measure of the actual behavior. Qualitatively, the sample-to-sample fluctuations of the MFPT increase as the trajectories start closer to the absorbing boundaries. However, this is not always true, as can be observed in Fig. 4(c) for the pie wedge with $\Theta=\pi / 3$ for which the light blue region extends toward the vertex of the wedge.

\section{CIRCULAR DOMAIN WITH APERTURE}

We now turn our attention to the first passage time problem of a Brownian particle in a circular domain of unit radius and the following boundary conditions: The segment with $|\theta|<\Theta / 2$ is absorbing while the remaining part of the outer circle is reflective. The aperture of the circular domain is thus of angle $\Theta$. One often encounters a three-dimensional version of this problem in cellular biochemistry, when one is interested in the time needed for a particle (a ligand, etc.), diffusing within a bounded domain (for instance, a microvesicle) to reach a small escape window or a binding site, which is an aperture in an otherwise reflecting boundary. This is the so-called narrow escape time problem, which has attracted considerable attention within the last two decades (see, e.g., Refs. [31,48] and references therein).

We analyze the shape of the uniformity distribution $P(\omega)$ as a function of the starting point of a Brownian walker. As in the previous section, we generate $N=10^{5}$ random walks commencing from the same starting position $\left(\rho_{0}, \theta_{0}\right)$ inside the unit circle and determine the set $\left\{\tau_{i}\right\}$ of first passage times to the location of the aperture. From these data we obtain $P(\omega)$ and compute the parameter $\chi$. In Fig. 5 we show the phase chart for the shape of $P(\omega)$ for four different sizes of the aperture: $\Theta=$ $\pi / 18, \pi / 2, \pi$, and $2 \pi$. Each symbol in the charts is light blue, beige, or dark blue, depending on whether the corresponding starting position leads to a bell-shaped, uniform, or M-shaped distribution $P(\omega)$, respectively. Note that the case shown in Fig. 5(d) reduces to a one-dimensional problem (see, e.g., Ref. [15]).

Similarly to our findings for the pie-wedge domain, we observe that the MFPT is not always a representative measure for the two-dimensional narrow escape time problem. Interestingly, the subdomain in which the MFPT is the least probable outcome (dark blue coding) is practically the same for small holes of $\Theta \leqslant \pi / 2$. It is worthwhile noting that while in this region $P(\omega)$ is always bimodal, the height of its maxima increases [and its value $P(\omega=1 / 2)$ representative
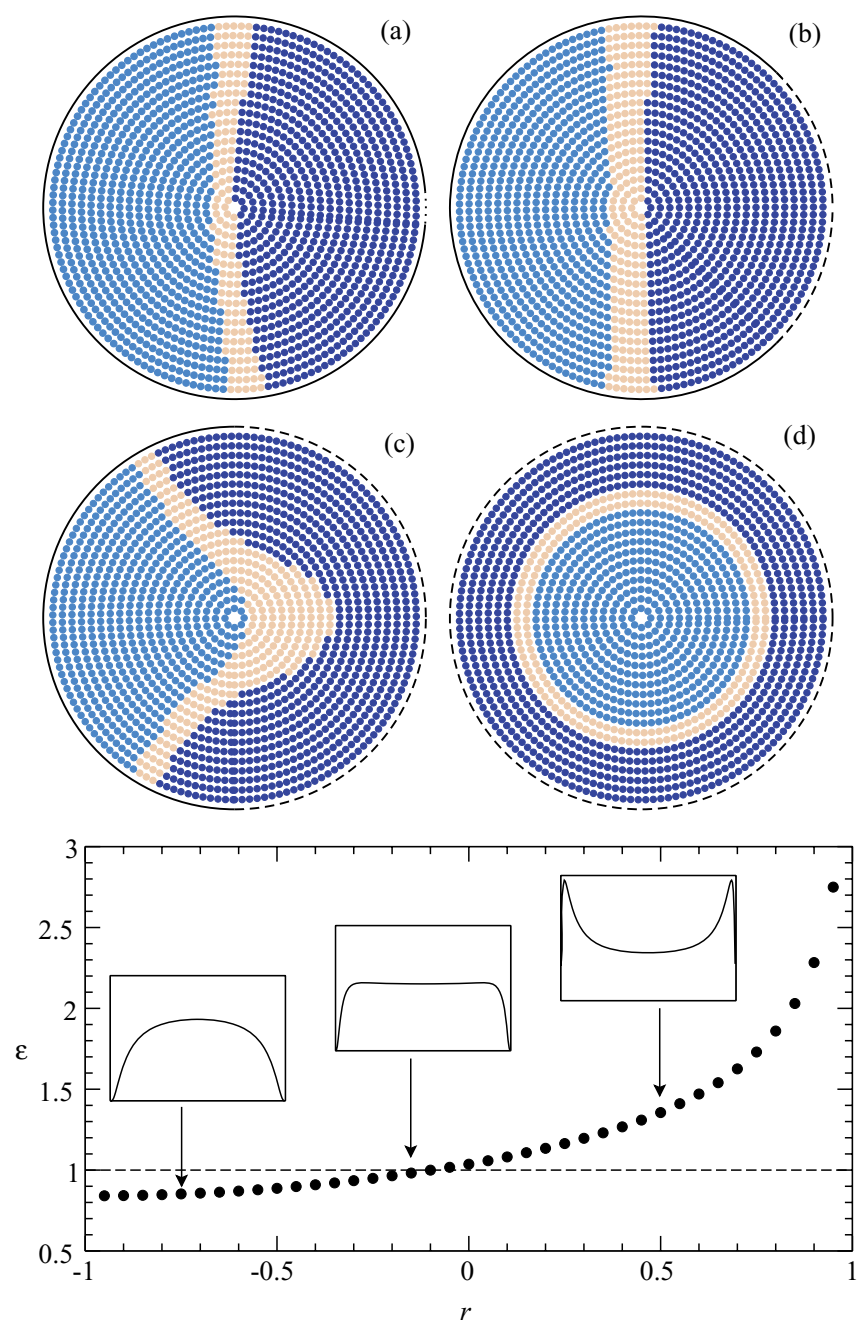

FIG. 5. (Color online) Phase chart for the shape of the uniformity distribution $P(\omega)$ for a Brownian walker in the unit circle with reflective BCs (solid lines) and an aperture of size $\Theta$ (absorbing BC, dashed lines). (a) $\Theta=\pi / 18$, (b) $\Theta=\pi / 2$, (c) $\Theta=\pi$, and (d) $\Theta=2 \pi$. Starting locations are colored light blue if $\chi<-\chi_{\star}$, dark blue if $\chi>\chi_{\star}$, and beige if $|\chi|<\chi_{\star}$, where $\chi_{\star}=1$. In the lower panel, the relative error of the FPT $\varepsilon$ is shown as a function of the initial radial position $r$ along the horizontal diameter of the unit circle with $\Theta=\pi / 2$, panel (b): $r=0$ is associated with the center of the circle and the point $r=1$ lies over the absorbing boundary.

of the MFPT decreases] depending on the distance from the opening.

In addition, from the set of first passage times $\left\{\tau_{i}\right\}$ we directly computed the MFPT and the variance $\operatorname{var}(\tau)$. Both statistical indicators grow with the distance from the absorbing boundary. A more sensitive measure is the relative error $\varepsilon$, defined as the ratio

$$
\varepsilon=\frac{\sqrt{\operatorname{var}(\tau)}}{\langle\tau\rangle}
$$

In the lower panel of Fig. 5 we show the dependence of the relative error on the starting position $\mathbf{r}_{0}$ for trajectories initiating along the symmetry axis of the domain, namely with respect to $r_{0}$ for fixed $\theta_{0}=0$. In agreement with the qualitative results of the phase chart, $\varepsilon<1$ only when $P(\omega)$ is bell shaped, 

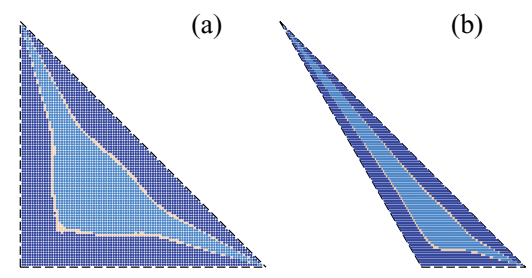

(b)

(c)

FIG. 6. (Color online) Phase chart of the shape of the uniformity distribution $P(\omega)$ for symmetric triangles with central angle $\pi / 2$ (a) and $2 \pi / 3$ (c), and for the asymmetric triangle with angles $2 \pi / 3, \pi / 4, \pi / 12$ (b). The color of the symbols is light blue if $\chi<-\chi_{\star}$, dark blue if $\chi>\chi_{\star}$, and beige if $|\chi|<\chi_{\star}$, with $\chi_{\star}=0.25$.

and the MFPT is the most probable outcome of a single-particle trajectory, namely, for trajectories starting far enough from the absorbing boundary. Clearly, the closer the starting position is to the absorbing boundary the larger the relative error becomes. Very near the absorbing boundary the standard deviation of the first passage time becomes much larger than its mean. We note that this result is generic irrespectively of the aperture size.

However $\varepsilon$ is just a number and it is not clear how to interpret it. For instance, $\varepsilon=1$ or $\varepsilon=2$ : Are these values too small or large enough to allow us to say that trajectory-totrajectory fluctuations are significant? On the other hand, the distribution of the simultaneity index which we discuss here gives a lucid answer on this question as manifested by the change of modality of $P(\omega)$.

\section{TRIANGULAR DOMAIN WITH ABSORBING BOUNDARIES}

Finally, as a complementary example we consider a domain whose boundaries are completely absorbing. We consider the triangular domains shown in Fig. 6: two symmetric triangles with central angle $\pi / 2$, panel (a), and $2 \pi / 3$, in panel (c), and an asymmetric triangle with angles $2 \pi / 3, \pi / 4, \pi / 12$ shown in panel (b).

In Fig. 6 we show the phase chart of the shape of $P(\omega)$; the results obtained are qualitatively the same as for the previous two examples with mixed boundary conditions. $P(\omega)$ is bell shaped and the MFPT the most probable outcome only when the trajectory starts far enough from the boundary. Also, similarly to what we observed for the pie-wedge domain, the domain in which $P(\omega)$ is unimodal extends toward the absorbing boundary if the vertex angle is less than $\pi / 2$.

\section{CONCLUSIONS}

We explored the problem of first passage of a Brownian particle to the absorbing boundary of finite, two-dimensional domains. From our study of the characteristic shapes of the associated distribution of the uniformity index $\omega$ we demonstrated that the MFPT represents the most probable outcome (and thus is quite meaningful) only if the trajectories start in a certain subregion of the total domain. For starting points in the complementary region the MFPT becomes the least probable outcome, indicating very large sampleto-sample fluctuations. These observations are generically important for single trajectory analysis of first passage time processes.

We showed that the associated separation into bell-shaped and $\mathrm{M}$-shaped forms of the uniformity distribution $P(\omega)$ is a robust property of Brownian motion by studying the problem in different symmetric and asymmetric domains with mixed or fully absorbing boundaries. We found that in general, sampleto-sample fluctuations of the first passage time increase when the trajectories start close to the target boundary, leading to the unexpected conclusion that in such situations the MFPT yields insufficient information, particularly if the absorption time is extracted from the outcome of very few single-particle trajectories.

Next, it is worthwhile mentioning that in many interesting situations the starting positions of the trajectories are randomly distributed inside the finite domain. From such analysis the socalled global MFPT is usually derived; see, e.g., Ref. [31]. Here we found that averaging the associated uniformity distribution $P(\omega)$ over the domain,

$$
P_{\mathrm{av}}(\omega)=\int_{\mathcal{S}} P_{\mathbf{r}_{0}}(\omega) d \mathbf{r}_{0},
$$

attains a uniform shape, except near $\omega=0$ and $\omega=1$. This appears to be a general property of $P_{\mathrm{av}}(\omega)$ associated with the probability conservation, and leads to the unexpected conclusion that the global MFPT has little meaning in such situations.

As a final remark, we emphasize that the approach outlined here is not limited to first passage phenomena only, but can be quite generally applied to probe the significance of sample-to-sample fluctuations of arbitrary random variables having distributions for which all moments exist. Such distributions, as shown in our work, may appear $\omega$ broad, in the sense that the corresponding uniformity distribution $P(\omega)$ is bimodal, or, alternatively, $\omega$ narrow with unimodal $P(\omega)$. We recall that the variable $\omega$ has a very lucid physical meaning and its distribution can be determined if the parental distribution of the random variable is known. Indeed such sort of heterogeneity analysis has very recently started to be used to quantify sample-to-sample fluctuations in mathematical finances [36,37], chaotic systems [38], analysis of distributions of the diffusion coefficient of proteins diffusing along DNAs [49], and FPT phenomena [15].

\section{ACKNOWLEDGMENTS}

The authors wish to thank Olivier Bénichou and Satya Majumdar for helpful discussion. The authors acknowledge financial support from the European Science Foundation and the hospitality of NORDITA, Stockholm, where part of this work was performed during the Non-Equilibrium Statistical Mechanics program. The research of T.G.M., R.M., and G.O. is partially supported by a Marie Curie International Research Staff Exchange Scheme Fellowship PIRSES-GA2010-269139 within the 7th European Community Framework Programme. R.M. acknowledges funding from the Academy of Finland within the FiDiPro program. The authors are partially supported by the ESF Research Network "Exploring the Physics of Small Devices." 
[1] C. Loverdo, O. Bénichou, M. Moreau, and R. Voituriez, Nat. Phys. 4, 134 (2008).

[2] C. Loverdo, O. Bénichou, M. Moreau, and R. Voituriez, J. Stat. Mech. (2009) P02045.

[3] O. Bénichou et al., Nat. Chem. 2, 472 (2010).

[4] O. Bénichou, M. Moreau, and G. Oshanin, Phys. Rev. E 61, 3388 (2000).

[5] T. G. Mattos and Fábio D. A. Aarão Reis, J. Chem. Phys. 131, 014505 (2009).

[6] M. von Smoluchowski, Z. Phys. Chem. 92, 129 (1917).

[7] G. L. Gerstein and B. B. Mandelbrot, Biophys. J. 4, 41 (1964).

[8] A. N. Burkitt, Biol. Cybern. 95, 1 (2006).

[9] G. M. Viswanathan et al., Nature (London) 401, 911 (1999).

[10] G. M. Viswanathan et al., The Physics of Foraging: An Introduction to Random Searches and Biological Encounters (Cambridge University Press, Cambridge, 2011).

[11] O. Bénichou, M. Coppey, M. Moreau, P. H. Suet, and R. Voituriez, Phys. Rev. Lett. 94, 198101 (2005); C. Loverdo, O. Bénichou, M. Moreau, and R. Voituriez, Phys. Rev. E 80, 031146 (2009); O. Bénichou, C. Loverdo, M. Moreau, and R. Voituriez, Rev. Mod. Phys. 83, 81 (2011).

[12] M. A. Lomholt, T. Ambjörnsson, and R. Metzler, Phys. Rev. Lett. 95, 260603 (2005); M. A. Lomholt, T. Koren, R. Metzler, and J. Klafter, Proc. Natl. Acad. Sci. USA 105, 11055 (2008); M. A. Lomholt et al., ibid. 106, 8204 (2009).

[13] G. Oshanin, H. S. Wio, K. Lindenberg, and S. F. Burlatsky, J. Phys.: Condens. Matter 19, 065142 (2007); J. Phys. A 42, 434008 (2009); G. Oshanin, O. Vasilyev, P. Krapivsky, and J. Klafter, Proc. Natl. Acad. Sci. USA 106, 13696 (2009).

[14] F. Rojo, C. E. Budde, and H. S. Wio, J. Phys. A 42, 125002 (2009); F. Rojo et al., ibid. 43, 345001 (2009).

[15] C. Mejia-Monasterio, G. Oshanin, and G. Schehr, J. Stat. Mech. (2011) P06022.

[16] J. M. Newby and P. C. Bressloff, J. Stat. Mech. (2010) P04014; P. C. Bressloff and J. M. Newby, Phys. Rev. E 85, 031909 (2012).

[17] I. G. Portillo, D. Campos, and V. Méndez, J. Stat. Mech. (2011) P02033.

[18] M. R. Evans and S. N. Majumdar, Phys. Rev. Lett. 106, 160601 (2011); J. Phys. A 44, 435001 (2011).

[19] E. Gelenbe, Phys. Rev. E 82, 061112 (2010).

[20] A. L. Lloyd and R. M. May, Science 292, 1316 (2001).

[21] A. Hanke and R. Metzler, J. Phys. A 36, L473 (2003); H. C. Fogedby and R. Metzler, Phys. Rev. Lett. 98, 070601 (2007).

[22] G. Oshanin, J. Klafter, and M. Urbakh, Europhys. Lett. 68, 26 (2004); J. Phys.: Condens. Matter 17, S3697 (2005).

[23] V. Palyulin and R. Metzler, J. Stat. Mech. (2012) L03001.

[24] J. P. Bouchaud and M. Potters, Theory of Financial Risk and Derivative Pricing: From Statistical Physics to Risk Management (Cambridge University Press, Cambridge, 2003).

[25] K. Lindenberg and B. J. West, J. Stat. Phys. 42, 201 (1986).

[26] S. Redner, A Guide to First-Passage Processes (Cambridge University Press, Cambridge, 2001).
[27] A. V. Chechkin et al., J. Phys. A 36, L537 (2003); T. Koren, M. A. Lomholt, A. V. Chechkin, J. Klafter, and R. Metzler, Phys. Rev. Lett. 99, 160602 (2007).

[28] R. Metzler and J. Klafter, Physica A 278, 107 (2000); H. Scher et al., Geophys. Res. Lett. 29, 1061 (2002).

[29] S. Condamin et al., Nature (London) 450, 77 (2007).

[30] S. Condamin, O. Bénichou, and M. Moreau, Phys. Rev. Lett. 95, 260601 (2005); Phys. Rev. E 75, 021111 (2007).

[31] B. Meyer, C. Chevalier, R. Voituriez, and O. Bénichou, Phys. Rev. E 83, 051116 (2011); C. Chevalier, O. Bénichou, B. Meyer, and R. Voituriez, J. Phys. A 44, 025002 (2011).

[32] J.-H. Jeon, A. V. Chechkin, and R. Metzler, Europhys. Lett. 94, 20008 (2011).

[33] S. N. Majumdar, Current Science 77, 370 (1999).

[34] G. Oshanin and S. Redner, Europhys. Lett. 85, 10008 (2009).

[35] M. Grabchak and G. Samorodnitsky, Quantitative Finance 10, 883 (2010).

[36] G. Oshanin and G. Schehr, Quantitative Finance 12, 1325 (2012).

[37] G. Oshanin, Yu. Holovatch, and G. Schehr, Physica A 390, 4340 (2011).

[38] C. Mejía-Monasterio, G. Oshanin, and G. Schehr, Phys. Rev. E 84, 035203 (2011).

[39] G. Oshanin, A. Mogutov, and M. Moreau, J. Stat. Phys. 73, 379 (1993); G. Oshanin, S. F. Burlatsky, M. Moreau, and B. Gaveau, Chem. Phys. 177, 803 (1993); C. Monthus, G. Oshanin, A. Comtet, and S. F. Burlatsky, Phys. Rev. E 54, 231 (1996).

[40] T. G. Mattos, C. Mejía-Monasterio, R. Metzler, and G. Oshanin (unpublished).

[41] I. Eliazar, Physica A 356, 207 (2005).

[42] I. Eliazar and I. M. Sokolov, Physica A 391, 3043 (2012).

[43] I. Eliazar and I. M. Sokolov, J. Phys. A 43, 055001 (2010).

[44] I. M. Sokolov and I. I. Eliazar, Phys. Rev. E 81, 026107 (2010).

[45] H. S. Carslaw and J. C. Jaeger, Conduction of Heat in Solids (Oxford University Press, Oxford, 1959).

[46] A. Metzler, Stat. Prob. Lett. 80, 277 (2010).

[47] B. D. Hughes, Random Walks and Random Environments (Oxford University Press, Oxford, 1995).

[48] M. J. Ward and J. B. Keller, SIAM J. Appl. Math. 53, 770 (1993); I. V. Grigoriev, Y. A. Makhnovskii, A. M. Bereshkovskii, and V. Y. Zitserman, J. Chem. Phys. 116, 9574 (2002); Y. Levin, M. A. Idiart, and J. J. Arenzon, Physica A 354, 95 (2005); O. Bénichou and R. Voituriez, Phys. Rev. Lett. 100, 168105 (2008); G. Oshanin, M. Tamm, and O. Vasilyev, J. Chem. Phys. 132, 235101 (2010); O. Benichou, D. Grebenkov, P. Levitz, C. Loverdo, and R. Voituriez, Phys. Rev. Lett. 105, 150606 (2010); A. Singer, Z. Schuss, D. Holcman, and R. S. Eisenberg, J. Stat. Phys. 122, 437 (2006); A. F. Cheviakov, M. J. Ward, and R. Straube, Multiscale Model. Simul. 8, 836 (2010).

[49] D. Boyer, D. S. Dean, C. Mejia-Monasterio, and G. Oshanin, Phys. Rev. E 85, 031136 (2012). 